\title{
Enseñanza de las ideas principales: problemas en el paso de la teoría a la práctica
}

\section{Nuria Carriedo y Jesús Alonso}

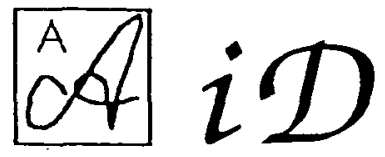

Uno de los sueños de todo educador es encontrar conceptos o métodos que sean a la vez poderosos y extremadamente simples. La «idea principal» se ha presentado como una de estas panaceas que permite construir estrategias de comprensión lectora adecuadas a los relativamente complejos modelos cognitivos, mediante un procedimiento de atractiva sencillez. En este artículo se aborda uno de los problemas más importantes y menos tratados de este prometedor enfoque. La propia denominación de «la idea principal» supone la existencia de un única idea principal y la univocidad de ésta: todo el mundo convendrá en una única y misma idea, la del maestro será la misma a la de cada uno de los alumnos.

El problema planteado por los autores de que este supuesto no se realiza (bay divergencias fuertes en la identificación de la idea) abre vías menos simplistas pero quizá más atractivas a la enseñanza desde «la idea principal».

\section{INTRODUCCION}

En los últimos años hemos asistido a una gran proliferación de programas de instrucción en comprensión lectora que - con mayor o menor éxitopretendían que los chicos llegaran a comprender la información más importante del texto.

La investigación sobre este tipo de programas instruccionales se ha incrementado de manera muy rápida. Sin embargo, esto no se ha visto acom- 
pañado por la clarificación de lo que constituye el concepto de idea principal del texto ni de los procesos cognitivos implicados en su identificación. Esta pueda ser una de las razones por las que, pese a la gran proliferación de programas, los resultados no son todo lo concluyentes que sería deseable.

Cunningham y Moore (1986), en un excelente trabajo, citan hasta once tipos de cuestiones diferentes para preguntar por lá información más importante del texto y hasta treinta y seis estrategias distintas relacionadas con la instrucción en este tema y que han aparecido citadas frecuentemente en revistas de Psicología desde 1920 hasta 1940.

La situación ha mejorado sólo parcialmente a partir de los años 70 con el desarrollo de modelos de estructura del discurso, que permiten identificar de una forma empírica, lo que constituye la información relevante del texto (e.g., Meyer, 1975; Kintsch y Van Dijk, 1978; Mandler y Johnson, 1977; etc.). Sin embargo, decimos que sólo ha mejorado parcialmente, porque si bien suponen un intento de operativizar el concepto de idea principal asimilándolo al de macroestructura textual, este último concepto sigue siendo parcialmente ambiguo no sólo para los sujetos a los cuales se instruye, sino también para los propios instructores: investigadores y profesores.

Para los investigadores sigue resultando ambiguo porque a pesar de este intento de operativización del constructo, en la literatura sigue habiendo una gran confusión terminológica, o lo que es lo mismo, parafraseando a Winograd y Bridge (1986) «los investigadores utilizan las mismas etiquetas, pero están examinando diferentes constructos». Estos mismos autores llegan a identificar hasta diez términos que los investigadores utilizan frecuente e indistintamente para hacer referencia a la información importante, a saber, idea principal, esencia, resumen, puntos principales, proposiciones supraordenadas, tema, tópico, estructura de texto, macroestructura y superestructuras esquemáticas.

De forma paralela, el estudio ya citado de Cunningham y Moore revela que el concepto también es ambiguo para los sujetos y para los profesores. Estos autores llegan a identificar - a través de lo que los alumnos responden cuando se les pregunta por la idea principal de un texto- nueve categorías que hacen referencia a la información relevante, a saber: esencia, interpretación, palabra clave, resumen o diagrama selectivo, tema, título, tópico, etiqueta, frase tópica y frase temática. Todas estas categorías se caracterizan porque aportan cierta información sobre el texto, y según ellos son igualmente legítimas. Además encontraron que estas nueve categorías también aparecían entre los profesores y entre los futuros profesores.

Partiendo de esta situación, algunos autores han cuestionado la efectividad de la instrucción en ideas principales debido por un lado a la confusión terminológica, y por otro a la falta de transferencia de lo aprendido a textos más naturales. Nuestro propósito en este momento no es cuestionar este tipo de instrucción, sino establecer las coordenadas que creemos necesarias para que tenga éxito, sobre todo, si partimos de que el propósito fundamental de la misma es el de facilitar el aprendizaje de los sujetos.

Además de los conceptos de lectura (y más específicamente de idea principal) que posean los sujetos, los profesores y los propios investigadores, otros factores, como son el tipo de textos utilizados y la situación instruccional, son también relevantes y deberían ser considerados a la hora de la instrucción. Sin embargo, en el desarrollo de los diferentes programas de entrenamiento no se han controlado cada uno de estos factores. 
Hasta el momento, gran parte de los programas de instrucción en comprensión de ideas principales que se han realizado, han ido dirigidos a enseñar a los sujetos del ciclo medio o superior a identificar la idea principal o a hacer resúmenes selectivos de los textos (e.g., nuestro país, Vidal Abarca, 1989; Sánchez, 1990).

La forma de llevar a cabo estos programas consistía en que el mismo investigador o algún colaborador de éste se trasladaba al centro escolar un día o dos a la semana y en una de las clases instruía sobre comprensión de textos. Además, para realizar la instrucción, se utilizaban adaptaciones de los textos escolares pero no los libros de texto como tales y en algunos casos, se trabajaba con un número de sujetos bastante reducido. Los resultados obtenidos, en general, fueron satisfactorios, pero no homogéneos ni consistentes (e.g., no hay correlación entre las puntuaciones de los sujetos en distintas pruebas utilizadas para valorar el programa, o mejoran más unos sujetos que otros).

Si analizamos el peso que tienen cada uno de los factores enunciados en los programas de entrenamiento, vemos que la mayoría van dirigidos a escolares, que están diseñados por investigadores ajenos a la escuela en cuestión, que se realizan o bien fuera del horario escolar, o bien dentro pero no por el propio profesor, que el entrenamiento no se incluye dentro de lo que constituye la clase curricular (se entrena algún día a la semana y de forma descontextualizada) y que se utilizan textos del mismo nivel escolar, pero adaptados, cuando en realidad los textos que tienen que estudiar los escolares son los que aparecen en sus libros de texto.

Para justificar la falta de contextualización de este tipo de entrenamiento tanto con respecto a los textos empleados como a quién debe realizar la instrucción, la mayoría de los investigadores han argumentado por una parte, que los libros de textos más comúnmente utilizados son estructuralmente muy complejos (Magnus y Hare, 1986; Hare, Rabinowitz y Schieble, 1989) y por lo tanto son difícilmente utilizables para enseñar a identificar la idea principal a partir de la estructura de los textos; y por otra, si bien algunos han reconocido que este tipo de instrucción debería realizarse por los propios profesores, no reconocen sin embargo, la necesidad de entrenar a estos últimos (e.g., Sánchez et al., 1990).

Finalmente, otro aspecto a considerar para valorar la eficacia de la instrucción en idea principal, es el problema de la evaluación.

En este sentido, se nos plantean una serie de interrogantes: ¿qué es lo que realmente se evalúa cuando se habla de idea principal?, ¿qué evalúan los distintos programas de entrenamiento en comprensión de textos? y finalmente ¿qué implicaciones tiene la evaluación?

Como ya hemos mencionado a lo largo de esta introducción, los procedimientos de entrenamiento que se han desarrollado dirigidos a la mejora de la comprensión de textos, no han sido todo lo eficaces que se esperaba. Esto puede deberse, como hemos argumentado, a la confusión terminológica y conceptual en torno a la información importante de los textos, pero también creemos que la confirmación de la eficacia de los distintos programas de entrenamiento pasa por el problema de la evaluación (Alonso et al., 1987, 1990).

La mayoría de las investigaciones dirigidas a entrenar comprensión de textos han utilizado de forma preferente el método de evaluación de Meyer 
$(1975,1985)$, que analiza el recuerdo de lo textos mediante su análisis proposicional. No es nuestro propósito cuestionar este método de evaluación; sin embargo, sí queremos señalar que la elevada complejidad de su desarrollo puede ser la razón por la cual nos se han realizado esfuerzos por aplicar este método de análisis del recuerdo a otros textos diferentes al empleado por Meyer («los superpetroleros»). El resultado de esto es que sólo en nuestro país el texto de los superpetroleros se ha utilizado indistintamente, tanto para evaluar el recuerdo de niños de segunda etapa $\left(6 .^{\circ}\right.$ y $7 .^{\circ}$ de EGB), como para evaluar el recuerdo de niños de Bachillerato (Sánchez, 1990b; León, 1989).

Esto supone, en primer lugar, que -salvo excepciones-, no se ha intentado adaptar la técnica a los sujetos sino los sujetos a la técnica de que se disponía; $y$, en segundo lugar, que se olvida que la comprensión (desde el modelo teórico del cual parten la mayoría de estos programas, el interactivo), es el producto - entre otros factores - de la interacción entre los conocimientos previos del lector y las características del texto. Pues bien, indudablemente, no puede tener el mismo conocimiento del tema un niño de $6 .^{\circ}$ de EGB, que un niño de $1 .^{\circ}$ de BUP, por lo que la utilización de esta técnica de forma indiscriminada no queda justificada.

Por otra parte, nos parece insuficiente la utilización únicamente de una prueba de recuerdo para medir el impacto de un programa de entrenamiento sobre comprensión. Indudablemente, si nuestro propósito, como venimos mencionando, es facilitar el aprendizaje, una prueba de recuerdo es adecuada para medir la información que han retenido los niños $y$, por lo tanto, es necesaria; pero no suficiente, fundamentalmente porque esto supondría equiparar el recuerdo producido con la comprensión real del texto. En este sentido, estamos de acuerdo en que posiblemente se recuerda mejor aquello que se ha comprendido, y por lo tanto se pueden inferir muchas cosas sobre la comprensión a partir de lo recordado, pero... ¿estamos en condiciones de afirmar que lo contrario también es cierto, es decir, que los niños que recuerdan menos es porque comprenden menos?

Johnston (1983) afirma que "la capacidad de comprensión lectora y de producción (recuerdo) no se correlacionan perfectamente" (p. 82) y para apoyarlo analiza las demandas cognitivas de una tarea de recuerdo libre. Para realizar una tarea de este tipo, un lector cuenta con unas determinadas habilidades de producción (las cuales no son las mismas para todos los sujetos), y además debe conocer el nivel de detalles que debe retener y producir, el grado en que debe mantener la estructura superficial del texto original, debe ser capaz de recuperar la información —entendida y almacenada previamente- en el momento en que sea preciso, debe decidir en qué punto comenzar la recuperación y cómo buscar la información, almacenada, y finalmente, debe tener en cuenta la perspectiva del examinador para decidir cómo presentará lo que recuerda. Todo esto, por supuesto, depende de que cl sujeto esté motivado para la realización de la tarea. En la práctica -como el mismo Jonhston ilustra - se pueden obtener protocolos de recuerdo idénticos correspondientes a diferentes procesos cognitivos.

La necesidad de valorar la efectividad de los diferentes programas de comprensión de textos debe tener en cuenta tanto las características del proceso que evaluamos, como del proceso de evaluación en sí mismo, es decir, es necesario investigar sobre los aspectos que deseamos medir, sobre cómo 


\section{1}

medirlos y sobre los factores que influyen en la actuación del individuo. Sin embargo, tal y como señala Jonhston, «la evalución actual de la comprensión lectora no es satisfactoria en parte por las limitaciones que sufre, y en parte, por intereses teóricos contrapuestos» (p. 89).

Nuestro propósito al escribir un artículo como éste es múltiple:

1. Mostrar la necesidad de entrenar a los escolares en la comprensión de textos y en especial en lo relativo a la idea principal.

2. Mostrar la necesidad de definir lo más operativamente posible el concepto de idea principal por parte de los investigadores.

3. Mostrar la necesidad de entrenar a los profesores en comprensión de textos.

4. Mostrar la relevancia de la evaluación en el desarrollo de programas de este tipo.

\section{PRIMER ESTUDIO}

\section{¿Qué entiende un alumno cuando se le pregunta por la idea principal del texto?}

Este estudio tuvo como propósito realizar una réplica del realizado por Conningham y Moore $(1984,1986)$, para comprobar con una muestra española, si aparecían las mismas categorías de idea principal que ellos habían identificado $\mathrm{y}$ de esta forma justificar la necesidad de instruir a los alumnos en este tipo de habilidades.

La réplica del estudio se justifica por varias razones: en primer lugar, porque se han realizado en nuestro país numerosos programas de entrenamiento sin haber sondeado antes si tal necesidad estaba presente en nuestra población de estudiantes; y en segundo lugar, porque los investigadores españoles frecuentemente importamos modelos norteamericanos sin hacer el esfuerzo de estudiar si estos modelos pueden aplicarse a nuestra población. Consideramos que en el tema que estamos abordando este último aspecto es especialmente importante porque las tradiciones instruccionales de ambos países son bastante diferentes, lo que probablemente afecta al modo de responder los alumnos al entrenamiento y la evaluación.

\section{Muestra}

La muestra estaba constituida por 428 sujetos pertenecientes a los cursos de 6. $.^{\circ}, 7^{\circ}$ y $8 .^{\circ}$ de EGB de dos colegios de clase media. La mitad de la muestra pertenecía al Colegio Juan XXIII de Alcobendas (Madrid) y la otra mitad pertenecía al Colegio Sagrado Corazón de Guadalajara.

\section{Procedimiento}

Se les presentaron a los sujetos 22 textos cortos naturales (no adaptados) de diferentes estructuras, extraídos de libros de texto existentes en el mercado español. A continuación se les pedía que leyeran el texto y que respondieran 
a la pregunta “¿Cuál es la idea principal de este texto?». Para la realización de la prueba dispusieron de todo el tiempo que necesitaron y se llevó a cabo dentro del horario escolar.

Para determinar cuáles de las respuestas dadas por los sujetos eran correctas, establecimos un sistema de categorías basándonos en el trabajo de Cunningham y Moore (1986), en el cual se identifican nueve tipos de idea principal, caracterizados todos ellos porque proporcionan información relevante acerca del texto. De estos nueve tipos, y tras un examen minucioso de las respuestas dadas por nuestros sujetos, no encontramos evidencia suficiente como para mantener todas las categorías, por lo que tuvimos que reducir el número de las mismas: eliminamos el resumen o diagrama selectivo, el tópico, la frase tópica y la frase temática. Las categorías quedaron de la siguiente forma: incorrecta, idea principal implícita, idea principal explícita, interpretación, título, tema y palabra clave.

A continuación ilustramos cada una de las categorías mostrando cómo utilizarlas para clasificar las respuestas de los sujetos dadas a uno de los textos presentados. La asignación de las respuestas a cada categoría fue realizada mediante acuerdo interjueces.

Texto: «Los volcanes y los terremotos, considerados hasta hace muy poco como fenómenos extraños e imprevistos, cobran hoy una importancia considerable de tres puntos de vista: científico, técnico y humano. En el plano científico, estos fenómenos tienen gran importancia para determinar la edad de las montañas o el proceso de formación del globo. En el aspecto técnico, puede significar el aprovechamiento de ingentes recursos del interior de la corteza por un mejor conocimiento de la misma. Finalmente, un aspecto que preocupa es la previsión de dichos fenómenos con el fin de paliar sus consecuencias catastróficas".

a. Idea Principal Implícita. Resumen de los contenidos explícitos del texto que contenga afirmaciones generales que sinteticen los contenidos específicos y prescinda de los contenidos redundantes.

En este caso, la idea principal está expresada explícitamente en el texto, y por tanto no existen respuestas clasificadas en ésta categoría.

b. Idea Principal Explícita. Frase aislada del párrafo o del texto que nos dice exactamente de lo que trata el texto o el párrafo.

«Cómo pueden beneficiarnos o perjudicarnos los volcanes y terremotos: pueden ayudarnos a saber algo más de la edad de la Tierra, se pueden aprovechar recursos naturales, pero destruyen hogares".

c. Interpretación. Generalización sobre la vida, el mundo, o el universo que el texto - como un todo- desarrolla, implica, o ilustra, pero no corresponde específicamente al tema del texto o a la palabra clave.

"Una cosa puede ser útil, aunque a veces sea fatal para la vida.»

d. Palabra Clave. Palabra o término que engloba el concepto aislado más importante del texto.

En nuestro estudio no se encuentran respuestas que pudiéramos incluir en esta categoría, no obstante, como ejemplo, podrían incluirse la palabra «volcanes" o "terremotos».

e. Título. Nombre dado al texto.

"Los volcanes y los terremotos."

f. Tema. Frase que engloba el tema o el contexto conceptual del texto pero sin revelar sus contenidos específicos. 
«Importancia y consecuencias de los volcanes y terremotos.»

g. Incorrecta. Incluimos en esta categoría todas aquellas respuestas que no nos daban ninguna información relevante sobre el texto.

Además de establecer las categorías de idea principal mencionadas anteriormente, también evaluamos el grado de adecuación de cada respuesta a su categoría de la siguiente forma:

- Se puntuaron con 2 aquellas respuestas que se adecuaban plenamente a la definición del tipo de idea principal correspondiente, y además estaban correctamente expresadas. (e.g. "Los volcanes y terremotos tienen importancia desde tres puntos de vista: científico, técnico y humano).

- Se puntuaron con 1 aquellas respuestas que, aun perteneciendo a uno de los tipos de idea principal definidos, estaban mal expresadas, eran incompletas o imprecisas en algunos de sus aspectos, o eran demasiado generales (e.g. «Los volcanes y terremotos son muy importantes desde tres puntos de vista»).

\section{Resultados}

En la Tabla 1 se presenta la clasificación de las respuestas dadas por los sujetos y el porcentaje de respuestas emitidas que corresponde a cada una de las categorías. La asignación de cada una de las respuestas de los sujetos a las categorías fue realizada por acuerdo interjueces $(r=0,90)$.

TABLA 1

\begin{tabular}{cllcccc}
\hline \multirow{2}{*}{ Elementos } & \multicolumn{5}{c}{ Tipos de respuesta* } \\
\cline { 2 - 7 } & 1 & 2 & 3 & 4 & 5 & 6 \\
\hline 1 & 52,8 & 7,9 & 3,3 & & & 26,4 \\
2 & 72 & 6,6 & 7 & & & 14,4 \\
3 & 57,5 & 0,9 & 15,4 & 0,5 & 1,4 & 24,2 \\
4 & 55,1 & & 19,2 & 0,9 & 0,5 & 24,3 \\
5 & 56,5 & & 9,8 & 1,8 & 0,5 & 31,3 \\
6 & 71 & 6 & 7,9 & & 0,5 & 14,5 \\
7 & 27,6 & 1,4 & 21,1 & 0,9 & & 50 \\
8 & 57,5 & 8,4 & 2,3 & 0,5 & & 31,3 \\
9 & 54,2 & 5,2 & 5,6 & 1,9 & & 32,7 \\
10 & 65,4 & 1,8 & 15,9 & & & 16,8 \\
11 & 59,3 & 3,3 & 12,6 & & & 25,2 \\
12 & 72,4 & & 5,6 & & & 21,9 \\
13 & 68,2 & 0,5 & 17,8 & 3,9 & & 9,8 \\
14 & 34,1 & 1,9 & 30,8 & 0,5 & 0,5 & 32,3 \\
15 & 54,7 & 0,5 & 16,8 & & & 28 \\
16 & 74,3 & 7 & 6,2 & & & 12,2 \\
17 & 56,1 & 2,3 & 11,7 & 0,9 & & 29 \\
18 & 56,1 & 1,8 & 10 & 0,5 & & 30,9 \\
19 & 72,4 & 16,8 & 3,8 & & & 7 \\
20 & 71,5 & 0,5 & 13,1 & 1,9 & & 13,1 \\
21 & 63,1 & 7,9 & 15,4 & 0,5 & & 13 \\
22 & 72,9 & 0,5 & 7,9 & 1,2 & & 17,7 \\
$x$ & 60,2 & 3,69 & 11,25 & 0,7 & 0,15 & 23,4 \\
\hline
\end{tabular}

*1. Incorrecta. 2. Interpretación. 3. Tema. 4. Título. 5. Palabra Clave. 6. Idea Principal. 
Discusión

Los resultados muestran que la mayoría de los sujetos de $6 .^{\circ}, 7 .^{\circ}$ y $8 .^{\circ}$ de EGB no saben lo que es la idea principal del texto (el $60,2 \%$ de las respuestas emitidas son incorrectas, es decir, no proporcionan ningún tipo de información relevante acerca del texto). Del porcentaje restante, el 23,4\% corresponde a la categoría de idea principal estrictamente hablando, mientras que $15,81 \%$ restante de las respuestas emitidas, si bien nos proporcionan información relevante sobre el texto, no constituyen estrictamente hablando la idea principal. En este punto estamos en desacuerdo con Cuningham y Moore (1986), ya que para estos autores todas las categorías de respuesta son igualmente válidas. Sin embargo, desde nuestro punto de vista, y partiendo del propósito anteriormente mencionado de facilitar el aprendizaje, consideramos que no tienen las mismas implicaciones las respuestas clasificadas en la categoría de idea principal que, por ejemplo, las clasificadas como título. En nuestro ejemplo, no es lo mismo - desde el punto de vista del aprendizajeque el chico responda ante la pregunta de cuál es la idea principal del texto con... "los volcanes y los terremotos», o con «Los volcanes y terremotos son muy importantes desde tres aspectos: científico, técnico y humano"...

\section{Conclusiones}

De los resultados obtenidos y, partiendo de que este estudio sólo es un aproximación descriptiva al concepto de idea principal, pueden extraerse algunas conclusiones importantes:

a. En primer lugar, es necesario el desarrollo de programas de entrenamiento que se centren en enseñar a los alumnos a identificar la idea principal de los textos expositivos y el mensaje central de los textos narrativos que aparecen en sus libros de texto. Esta afirmación se justifica porque la mayoría de nuestros sujetos no sabe cuál es la idea principal de los textos.

b. En segundo lugar, que estos programas de entrenamiento deben partir de una definición lo más precisa posible de lo que constituye la idea principal de un texto $y$, asimismo, diferenciarla de otros tipos de información relevante sobre el mismo (en especial del tema y de las interpretaciones), sobre todo, si nuestro objetivo es facilitar el aprendizaje. Esta afirmación se justifica porque, como han mostrado los datos, no todos los sujetos entienden lo mismo cuando se les pide la idea principal de los textos.

c. Finalmente, los investigadores deberían poner especial cuidado en el tipo de tareas que proponen para llegar a identificar la idea principal. En este sentido, "no es infrecuente encontrar programas de entrenamiento que utilizan la tarea "poner títulos a los párrafos» como un paso para llegar a identificar la idea principal de los textos, o para hacer resúmenes de los mismos. En este momento no es nuestro objetivo valorar la adecuación de este tipo de tareas, sin embargo sí queremos señalar, que si no se especifican con la suficiente claridad, pueden contribuir a aumentar la confusión con respecto a la idea principal, puesto que uno de los términos que se asocia «incorrectamente» a ella es el de título.

Por otra parte, además de la constatación empírica de la necesidad de instruir en la comprensión de la idea principal, cabe preguntarse por qué los sujetos responden como lo hacen ante la pregunta formulada, o lo que es 
lo mismo, qué es lo que determina que centren su atención sobre algunos aspectos del texto y no sobre otros. Frecuentemente se ha afirmado que el propósito de lectura, el conocimiento previo del lector y la organización que el autor hace de las ideas del texto, son los factores fundamentales que determinan el sentido de la atención del lector, es decir, hacia qué aspectos del texto va dirigida. Sin embargo, la mayoría de los programas instruccionales desarrollados se centran fundamentalmente en la estructura del texto olvidando el papel que los otros dos factores juegan en la comprensión. Un estudio como el que hemos realizado muestra, entre otras cosas, la necesidad de trabajar con un propósito mucho menos ambiguo que el que se deriva de la pregunta “¿Cuál es la idea principal de el texto?, o ¿cuál es la organización de las ideas que hace el autor?, o simplemente, ¿cuál es la relación predominante en el texto?

\section{ESTUDIO 2}

\section{¿Qué entiende un profesor cuando se le habla de idea principal de un texto?}

Como ya hemos comentado anteriormente, la mayoría de los programas instruccionales sobre las ideas principales han sido llevados a cabo por los propios investigadores en el aula. No obstante, recientemente se ha intentado que fuesen los propios profesores quienes realizaran la instrucción, aunque, cuando esto se ha realizado, se ha defendido el hecho de que los profesores no necesitan ser instruidos.

Nuestro objetivo, al realizar un estudio como éste, era el de determinar si los profesores tienen claro el concepto de idea principal, si saben qué tipo de estrategias deben de utilizar para enseñarla y si son conscientes de la importancia que tiene esta habilidad en el aprendizaje.

\section{Mutstra}

En el estudio han participado 11 profesores de las áreas de Ciencias Sociales, Ciencias Naturales y Lenguaje, de los cursos $6 .^{\circ}, 7 .^{\circ}$ y $8 .^{\circ}$ de EGB, procedentes de colegios de Alcalá de Henares (Madrid), distribuidos de la siguiente forma: dos profesoras del colegio público García Lorca, cinco del colegio público Beatriz Galindo, uno del colegio público Reyes Católicos y una del colegio privado Escolapias.

El número de años de docencia de los profesores oscila desde 3 (valor mínimo) hasta 29 (valor máximo), siendo la mediana de 20 años.

\section{Procedimiento}

Se les presentaron a los sujetos ocho textos expositivos de diferentes estructuras extraídos de la revista "Investigación y Ciencia».

Los textos se diferenciaban en cuanto a su contenido y en cuanto a su estructura:

a. Contenido: de los ocho textos presentados, cinco podían considerarse del área de Ciencias Naturales y tres del área de Ciencias Sociales.

b. Estructura: La asignación de cada uno de los textos a una estructura fue realizada mediante acuerdo interjueces. La razón de esto es que trabajamos con textos naturales, los cuales son estructuralmente más complejos, por lo 


\section{6}

que la determinación de la estructura retórica predominante en el texto puede suponer ciertas dificultades. Tras la discusión, los textos quedaron como sigue: uno de generalización, dos de causa-efecto, un enumerativo o de listado, uno secuencial, uno de problema-solución, uno comparativo y uno clasificativo'.

La tarea de los profesores consistía en leer cada uno de los textos y responder a la siguiente pregunta: ... «Si al término de una sesión de instrucción tuvieran que resumir a sus alumnos cuál es la idea principal del texto que acaban de leer. Qué les dirían?».

Para codificar las respuestas emitidas por los profesores, empleamos el mismo sistema que habíamos utilizado para categorizar las de los chicos.

\section{Resultados}

En la Tabla 2, se muestra la clasificación de las respuestas dadas por los profesores y el porcentaje de respuestas emitidas que corresponde a cada una de las categorías.

TABLA 2

\begin{tabular}{cccccc}
\hline \multirow{2}{*}{ Elementos } & \multicolumn{5}{c}{ Tipos de respuesta* } \\
\cline { 2 - 6 } & 1 & 2 & 3 & 4 & 5 \\
\hline 1 & 30 & 10 & 50 & & 6 \\
2 & 10 & & & & 10 \\
3 & 10 & & 20 & 70 \\
4 & 40 & & 10 & 50 \\
5 & 10 & & 10 & 80 \\
6 & 20 & 10 & 20 & 50 \\
7 & 40 & & 10 & 50 \\
8 & 20 & & & 80 \\
$\mathrm{x}$ & 22,5 & 2.5 & 15 & & 60 \\
\hline
\end{tabular}

* 1. Incorrecta. 2. Interpretación. 3. Tema. 4. Título. 5. Palabra Clave. 6. Idea Principal

\section{Discusión}

Los resultados muestran que la mayor parte de las respuestas emitidas por los sujetos pertenecen a la categoría que hemos denominado «idea principal» $(60 \%)$, y el $22,5 \%$ de las mismas pueden considerarse incorrectas. Del resto de las respuestas, el 2,5\% pertenecen a la categoría de interpretación, y el $15 \%$ a la de tema. En este estudio (tal y como cabía esperar), no han aparecido respuestas correspondientes a las categorías de palabra clave o de título.

Estos resultados muestran que el concepto de idea principal también resulta parcialmente ambiguo para los propios profesores. Esta afirmación está basada por una parte, en que no siempre saben cuál es la idea principal de un texto, y por otra en que este concepto se confunde con relativa frecuencia con el tema general del texto. Además, un aspecto que nos parece muy significativo es el hecho de que un $2,5 \%$ de las respuestas correspondan a interpretaciones. Teniendo en cuenta que hemos definido esta categoría 
como una «generalización sobre la vida, el mundo, o el universo que el texto - como un todo- desarrolla, implica, o ilustra, o lo que es lo mismo, una elaboración personal que el lector realiza a partir de la información del téxto pero que no tiene por qué corresponder con la idea principal», no deja de ser sorprendente que sea el propio profesor quien oriente a sus alumnos hacia este tipo de información y no especialmente hacia lo relevante desde un punto de la comprensión lectora.

\section{Conclusiones}

A partir del estudio realizado con los profesores, podemos extraer algunas conclusiones importantes. En primer lugar, pensamos que hay suficiente evidencia en los datos como para afirmar que si se pretende que sean los profesores quienes instruyan a sus alumnos en la comprensión de textos, es necesario que sean instruidos explícitamente en lo que consideramos la idea principal de un texto, diferenciándola especialmente del tema del texto y en menor medida, pero también, de las interpretaciones.

En segundo lugar, es necesario instruir a los profesores no sólo en QUE instruir (identificación de la idea principal), sino también en COMO hacerlo. Para justificar esto, en este mismo estudio, preguntamos a los profesores « ¿sobre qué aspectos del texto les dirían a sus alumnos que se fijasen para llegar a identificar la idea principal del texto?»². Pues bien, en otros estudios que estamos realizando en estos momentos, hemos constatado que incluso los profesores que habían identificado la idea principal del texto, no sabían sobre qué aspectos estructurales del mismo dirigir la atención de sus alumnos. Por ejemplo, para uno de los textos de causa-efecto, se obtuvieron indistinta y simultáneamente respuestas ...«sobre la comparación, sobre los grupos que establece el autor, sobre las consecuencias, sobre las causas», etc. Y aún más, cuando se les pedía a los profesores que explicaran o ejemplificaran cómo enseñarían a sus alumnos a identificar la idea principal para comprender el texto, muy pocos hicieron referencia a claves estructurales.

\section{Notas}

\footnotetext{
1 Además de las estructuras retóricas identificadas por Meyer (1975), hemos incluido tres más, que aparecen con frecuencia en los libros de texto: descripción-generalización, enumeración, comparación-contraste, clasificación, secuencia, causa-efecto, problema-solución y argumentación. Un ejemplo de las mismas puede encontrarse en J. Alonso (1990). Motivación, pensamiento y aprendizaje. Santillana (en prensa).

${ }_{2}$ Datos sin publicar de otro estudio realizado para la tesis doctoral Enseñar a Comprender. Desarrollo y valoración de un programa de entrenamiento dirigido a profesores para mejorar en sus alumnos la comprensión de textos escolares.
}

\section{Referencias}

Alonso. J. (En prensa). Motivación, pensamiento y aprendizaje. Madrid: Santillana.

Alonso, J., Carriedo, N. y GonzAlez, E. (En prensa). Evaluación de la comprensión lectora. Un estudio piloto sobre la identificación de las ideas principales y el resumen de textos.

Alonso, J., Mateos, M., Carrera, P. y Carriedo, N. (1987). Comprensión de la lectura: una aproximación al problema de la evaluación. Boletín del Instituto de Ciencias de la Educación, n. 11.

BaumanN, J. F. y Serra, J. K. (1984). The frecuency and placement of main ideas in children's social studies textbooks: A modified replication of Braddock's research on topic sentences. Journal of Reading Behavior, 16, 27-40. 
Cunningham, J. W. y Moore, D. W. (1986). The confused world of main idea. En J. F. Baumann (ed.), Teaching main idea comprehension (pp. 1-17). Newark, DE: International Reading Comprehension. (Trad. cast. Madrid: Visor, 1990)

Hare, V. C., Rabinowizt, M. y SChifble, K. M. (1989). Text effects of main idea comprehension. Reading Research Quarterly, XXIV (1), pp. 73-88.

JONHSTON, P. H. (1983). La evaluación de la comprensión lectora. Un enfoque cognitivo, Madrid: Visor, 1989.

KintsCh, W. y VAn Dijk, T. A. (1978). Toward a model of text comprehension and production. Psychological Review, 85, 363-394.

Mandi.er, J. M. y Johnson, N. S. (1977). Rememberance of things parsed: story structure and recall. Cognitive Psychology, 9, 111-151.

LEÓN. J. A. (1989). Comprensión y memoria de textos expositivos. Tesis doctoral sin publicar. Universidad Autónoma de Madrid.

Magnus, K. y HARE, V. C. (1986). The unintented road to ecological invalidity: Compromises in instrument construction. Comunicación en la Texas National Reading Conference, Austin.

MEYER, B. J. F. (1975). The organization of prose and its effects on memory. Amsterdam: NorthHolland.

MEYer, B. J. F. (1985). Prose analysis: purposes, procedures and problems. En B. K. Britton y J. B. Black (Eds.), Understanding expository text. Hillsdale, Nueva Jersey: Erlbaum.

SÁnchtz, E. (1990a). La comprensión de textos en el aula. Un programa para instruir en la comprensión de textos en la etapa secundaria obligatoria. Documentos Didácticos 1. Instituto de Ciencias de la Educación de la Universidad de Salamanca.

Sánchtz, E. (1990b). Estructuras textuales y procesos de comprensión. Estudios de Psicología, $41,21-40$.

VIDAL ABARCA. (1990). Un programa para la enseñanza de la comprensión de ideas principales de textos expositivos. Infancia y Aprendizaje, 49, pp. 53-73.

WINOGRAD, P. N. y BRIDGE, C. A. (1986). The comprehension of important information in written prose. En J. F. Baumann (ed.) Teaching main idea comprebension (pp. 1-17). Newark, DE: International Reading Comprehension.

\section{Enseñanza de las ideas principales: Problemas en el paso de la teoría a la práctica. $N$. Carriedo y J. Alonso. \\ CLEE, 1991, 9, pp. 97-108}

Resumen: En este artículo se hace una revisión del estado actual de la investigación sobre la enseñanza de las ideas principales desde el punto de vista teórico y aplicado: tanto en cuanto a los programas de entrenamiento como a las técnicas de evaluación. El análisis realizado, revela que tanto los investigadores, los profesores como los alumnos no tienen claro el concepto de lo que se considera idea principal de un texto, y esto tiene importantes repercusiones educativas. Para apoyar sus conclusiones, los autores presentan un estudio en el que se investiga el concepto de idea principal que tienen alumnos y profesores de $E G B$.

Datos sobre los autores: Nuria Carriedo es profesora del Departamento de Psicología Básica, Social y Metodología de la Universidad Autónoma de Madrid. Su área de investigación y trabajo gira en torno a la enseñanza de la comprensión de textos en el aula. Jesús Álonso Tapia es profesor de la Facultad de Psicología de la UAM y director de la Sección de Investigación del Instituto de Ciencias de la Educación de la UAM. Actualmente trabaja en motivación y comprensión lectora en el contexto escolar.

Dirección: Universidad Autónoma de Madrid, Facultad de Psicología. Campus de Cantoblanco, 28049 Madrid.

Agradecimientos: Queremos mostrar nuestro agradecimiento a los alumnos y profesores de los colegios Juan xxill de Alcobendas, Sagrado Corazón de Guadalajara, García Lorca, Beatríz Galindo, Reyes Católicos y Escolapias de Alcalá de Henares, por haber hecho posible este trabajo.

( ) de rodos los artículos. Deberá solicitarse por escrito autorización de CL\&E para el uso en forma de facsímil, fotocopia o cualquier otro medio de reproducción. CL\&E se reserva el derecho de interponer las acciones legales necesarias en aquellos casos en que se contravenga la ley de derechos de autor. 